\title{
CANONICAL HEIGHTS FOR PLANE POLYNOMIAL MAPS OF SMALL TOPOLOGICAL DEGREE
}

\author{
Mattias Jonsson AND Elizabeth Wulcan
}

\begin{abstract}
We study canonical heights for plane polynomial mappings of small topological degree. In particular, we prove that for points of canonical height zero, the arithmetic degree is bounded by the topological degree and hence strictly smaller than the first dynamical degree. The proof uses the existence, proved by Favre and the first author, of certain compactifications of the plane adapted to the dynamics.
\end{abstract}

\section{Introduction}

Silverman [Sil11] recently proposed a number of conjectures on the growth of heights and degrees under iterates of rational selfmaps of projective space, and proved them for monomial maps. Here we study the growth of heights for a large class of plane polynomial maps.

Consider a polynomial mapping $f: \mathbf{A}^{2} \rightarrow \mathbf{A}^{2}$ defined over the field $\overline{\mathbf{Q}}$ of algebraic numbers. The first dynamical degree $\lambda_{1}$ is defined by

$$
\lambda_{1}:=\lim _{n \rightarrow \infty}\left(\operatorname{deg} f^{n}\right)^{1 / n} .
$$

The second dynamical degree $\lambda_{2}$ is the number of preimages under $f$ of a general closed point in $\mathbf{A}^{2}$. It follows from Bézout's Theorem that $\lambda_{2} \leq \lambda_{1}^{2}$. Following Guedj [Gue02] we say that $f$ has small topological degree if $\lambda_{2}<\lambda_{1}$.

Let $h$ be the standard logarithmic height on $\mathbf{P}^{2}(\overline{\mathbf{Q}}) \supseteq \mathbf{A}^{2}(\overline{\mathbf{Q}})$.

Main Theorem. Let $f: \mathbf{A}^{2} \rightarrow \mathbf{A}^{2}$ be a polynomial mapping of small topological degree, $\lambda_{2}<\lambda_{1}$. Then the limit

$$
\hat{h}:=\lim _{n \rightarrow \infty} \lambda_{1}^{-n} h \circ f^{n}
$$

exists, and is finite, pointwise on $\mathbf{A}^{2}(\overline{\mathbf{Q}})$. We have $\hat{h} \not \equiv 0$ and $\hat{h} \circ f=\lambda_{1} \hat{h}$. Further, if $P \in \mathbf{A}^{2}(\overline{\mathbf{Q}})$ and $\hat{h}(P)=0$, then

$$
\limsup _{n \rightarrow \infty} h\left(f^{n}(P)\right)^{1 / n} \leq \lambda_{2}<\lambda_{1} .
$$

If $f$ is moreover a polynomial automorphism, then $\hat{h}(P)=0$ iff $P$ is periodic.

Received by the editors February 2, 2012.

1991 Mathematics Subject Classification. Primary: 37P30; Secondary: 11G50, 37P15.

Key words and phrases. Canonical height, dynamical degrees, polynomial mappings, compactifications, arithmetic dynamics. 
As in [Sil11] we call $\hat{h}$ the canonical height associated to $f$, whereas the left hand side of $\left(^{*}\right)$ is the arithmetic degree of the point $P$. Our Main Theorem says that for points of canonical height zero, the height along the orbit grows relatively slowly.

The related Conjecture 3 in [Sil11] states that $\hat{h}(P)>0$ whenever $P$ has Zariski dense orbit in $\mathbf{A}^{2}$. By the Main Theorem, this holds for polynomial automorphisms, but we have not been able to establish it for noninvertible polynomial mappings of small topological degree. For such maps, the locus $\hat{h}=0$ may contain points for which $h \circ f^{n}$ grows exponentially; see Section 3.4.

The basic arithmetic dynamics of plane polynomial automorphisms is well understood, thanks to work of Silverman [Sil94], Denis [Den95], Marcello [Mar00, Mar03], Kawaguchi [Kaw06, Kaw09], Lee [Lee09], Ingram [Ing11] and others. In particular, the Main Theorem above and Conjecture 3 in [Sil11] were already known ${ }^{1}$ for polynomial automorphisms $f$ with $\lambda_{1}(f)>1$. The existing proofs make use of the inverse map $f^{-1}$ and also rely crucially on the Friedland-Milnor classification [FM89], which shows that, up to conjugation, $f$ is a composition of generalized Hénon maps and in particular regular in the sense of [Sib99]. In fact, the two results above are true for regular polynomial automorphisms of any dimension, see [Sil11, Theorem 36].

For noninvertible maps, no algebraic classification is known. Instead we exploit a result by Favre and the first author [FJ11] which shows that maps of small topological degree always admit compactifications that are well adapted to the dynamics. We refer to Section 2.3 for a precise statement. Given such a compactification, we work locally with a given absolute value and estimate the growth of the local height under iteration. When the absolute value is Archimedean, this was essentially carried out in [FJ11, Section 7], adapting techniques from the early work on the complex Hénon map, see [Hub86, HO94, FM89, BS91, FS92].

The existence of a compactification adapted to the dynamics is proved in [FJ11] using the induced dynamics on a suitable space of valuations, see also [FJ04, FJ07, Jon12]. In order to address Silverman's Conjecture 3 in our setting, one would likely have to refine this valuative analysis, something which is beyond the scope of the present paper.

The ergodic theory of polynomial maps of small topological degree over the complex numbers is studied in detail in [DDG1, DDG2, DDG3]. It would be interesting to see if these results have non-Archimedean or arithmetic analogues.

The paper is organized as follows. In Section 2, we recall some facts concerning dynamical degrees and heights. We also state the key result from [FJ11] on the existence of a compactification adapted to the dynamics. The Main Theorem is proved in Section 3.

\section{Background}

Unless indicated otherwise, we work over the field $\overline{\mathbf{Q}}$ of algebraic numbers.

2.1. Admissible compactifications. We use the standard embedding $\mathbf{A}^{2} \hookrightarrow \mathbf{P}^{2}$ given in coordinates by $\left(x_{1}, x_{2}\right) \mapsto\left[1: x_{1}: x_{2}\right]$. Let $\mathcal{L}$ be the set of affine functions on $\mathbf{A}^{2}$.

\footnotetext{
${ }^{1}$ The existence of $\hat{h}$ as a limit rather than a lim sup seems to be new, however.
} 
Definition 2.1. [FJ11] An admissible compactification of $\mathbf{A}^{2}$ is a smooth projective surface $X$ together with a birational morphism $\pi: X \rightarrow \mathbf{P}^{2}$ that is an isomorphism above $\mathbf{A}^{2}$.

We can thus view $\mathbf{A}^{2}$ as a Zariski open subset of $X$. By the structure theorem for birational morphisms of surfaces, $\pi$ is a finite composition of point blowups, so the divisor $X \backslash \mathbf{A}^{2}$ has normal crossing singularities.

Let $\xi \in X \backslash \mathbf{A}^{2}$ be a closed point. The closure in $X$ of at least one of the curves $\left\{x_{i}=0\right\} \subseteq \mathbf{A}^{2}, i=1,2$, does not contain $\xi$ : this is true already when $X=\mathbf{P}^{2}$. Pick local coordinates $z_{1}, z_{2} \in \mathcal{O}_{X, \xi}$ such that $X \backslash \mathbf{A}^{2} \subseteq E_{1} \cup E_{2}$, locally at $\xi$, where $E_{i}=\left\{z_{i}=0\right\}$. Write $b_{i}=-\max _{\ell \in \mathcal{L}} \operatorname{ord}_{E_{i}}(\ell) \in \mathbf{Z}_{\geq 0}$, where $\operatorname{ord}_{E_{i}}(\ell)$ is the order of vanishing of $\ell$ along $E_{i}$. Note that $b_{i}=-\operatorname{ord}_{E_{i}}(\ell)$ for a general affine function $\ell \in \mathcal{L}$. Thus $b_{i}=0$, iff $E_{i} \cap \mathbf{A}^{2} \neq \emptyset$. We can write

$$
x_{i}=z_{1}^{-b_{1}} z_{2}^{-b_{2}} \psi_{i}
$$

where $\psi_{i} \in \mathcal{O}_{X, \xi}$ and where $\psi_{i}(\xi) \neq 0$ for at least one $i$.

Similarly, if $f: \mathbf{A}^{2} \rightarrow \mathbf{A}^{2}$ is any dominant polynomial mapping, then

$$
f^{*} x_{i}=z_{1}^{\operatorname{ord}_{E_{1}}\left(f^{*} \ell\right)} z_{2}^{\operatorname{ord}_{E_{2}}\left(f^{*} \ell\right)} \chi_{i}
$$

for a general affine function $\ell \in \mathcal{L}$, where $\chi_{i} \in \mathcal{O}_{X, \xi}$. In this case, we do not claim that $\chi_{i}(\xi) \neq 0$ for some $i$.

2.2. Degree growth. See [Gue02, FJ07, FJ11] for more details on what follows. Let $f: \mathbf{A}^{2} \rightarrow \mathbf{A}^{2}$ be a dominant polynomial mapping defined over $\overline{\mathbf{Q}}$. The degree $\operatorname{deg} f$ of $f$ is the degree of $f^{*} \ell$ for a general affine function $\ell \in \mathcal{L}$. Thus $\operatorname{deg} f=\max _{i} \operatorname{deg} f^{*} x_{i}$.

It is easy to see that $\operatorname{deg} f^{n+m} \leq\left(\operatorname{deg} f^{n}\right)\left(\operatorname{deg} f^{m}\right)$; hence the limit $\lambda_{1}:=\lambda_{1}(f):=$ $\lim _{n \rightarrow \infty}\left(\operatorname{deg} f^{n}\right)^{1 / n}$ exists. It is called the first dynamical degree of $f$. The second dynamical degree $\lambda_{2}:=\lambda_{2}(f)$ is the topological degree of $f$, i.e., the number of preimages of a general closed point in $\mathbf{A}^{2}$. Note that $\lambda_{i}\left(f^{n}\right)=\lambda_{i}(f)^{n}$. It follows from Bézout's Theorem that $\lambda_{2} \leq \lambda_{1}^{2}$. While the degree $\operatorname{deg} f$ depends on the choice of embedding $\mathbf{A}^{2} \hookrightarrow \mathbf{P}^{2}$, the dynamical degrees $\lambda_{i}(f)$ do not.

The degree growth sequence $\left(\operatorname{deg} f^{n}\right)_{n=0}^{\infty}$ of plane polynomial maps was studied in detail in [FJ07, FJ11]; see also [BFJ08]. In particular, we have

Theorem 2.2. [FJ07, Theorem A'.] If $\lambda_{1}>1$, then $\operatorname{deg} f^{n} \sim n^{l} \lambda_{1}^{n}$ as $n \rightarrow \infty$, where $l \in\{0,1\}$. Further, $l=0$ unless $\lambda_{2}=\lambda_{1}^{2}$ and $f$ is conjugate to a skew product.

2.3. Dynamical compactifications. The following result from [FJ11] plays a key role in the proof of the Main Theorem.

Theorem 2.3. Let $f: \mathbf{A}^{2} \rightarrow \mathbf{A}^{2}$ be a polynomial map of small topological degree: $\lambda_{2}<\lambda_{1}$. Then, for every $\varepsilon>0$ there exist an integer $n_{0} \geq 1$, an admissible compactification $X$ of $\mathbf{A}^{2}$ and a decomposition $X \backslash \mathbf{A}^{2}=Z^{+} \cup Z^{-}$into (possibly reducible) curves $Z^{+}, Z^{-}$without common components, such that the following properties hold.

(1) If $F$ is any irreducible component of $Z^{-}$and $\ell \in \mathcal{L}$ is a general affine function, then

$$
\operatorname{ord}_{F}\left(f^{n_{0} *} \ell\right) \geq\left(\lambda_{2}+\varepsilon\right)^{n_{0}} \operatorname{ord}_{F}(\ell) .
$$


(2) The extension $f^{n_{0}}: X \rightarrow X$ of $f^{n_{0}}$ as a rational map is regular at any point on $Z^{+}$.

(3) There exists a closed point $\xi_{+} \in Z^{+} \backslash Z^{-}$such that $f^{n_{0}}\left(Z^{+}\right)=\left\{\xi_{+}\right\}$and $f\left(\xi_{+}\right)=\xi_{+}$. Further, we are in one of the following two cases:

(a) $\lambda_{1} \notin \mathbf{Q}$, there are two irreducible components $E_{1}, E_{2}$ of $Z^{+}$containing $\xi_{+}$and, locally at $\xi_{+}$, we have $f^{*} E_{i}=a_{i 1} E_{1}+a_{i 2} E_{2}$, where $a_{i j} \in \mathbf{N}$, and the $2 \times 2$ matrix $\left(a_{i j}\right)$ has spectral radius $\lambda_{1}$;

(b) $\lambda_{1} \in \mathbf{N}$, there is a unique irreducible component $E$ of $Z^{+}$containing $\xi_{+}$, $f(E)=\left\{\xi_{+}\right\}$and, locally at $\xi_{+}$, we have $f^{*} E=\lambda_{1} E$.

The theorem above corresponds to [FJ11, Lemma 7.3]. ${ }^{2}$ The fact that we may assume $\lambda_{1} \notin \mathbf{Q}$ in case (a) follows from Proposition 2.5 and the proof of Theorem 3.1 in [FJ11].

Note that everything in [FJ11] is stated over the complex numbers, and the assertions in (a) and (b) are slightly more precise than what is written here: they give normal forms in (possibly transcendental) local coordinates, based on the work of Favre [Fav00]. However, the main analysis in [FJ11] is purely algebraic. When working over a general algebraically closed field of characteristic zero one obtains Theorem 2.3.

2.4. Absolute values. Consider an admissible compactification $X$ of $\mathbf{A}^{2}$. Let $K$ be a number field such that $X$ and the map $\pi: X \rightarrow \mathbf{P}^{2}$ are defined over $K$.

Let $M_{K}$ be the set of (normalized) absolute values on $K$. For each $v \in M_{K}$, let $K_{v}$ be the completion. Write $X(K)$ for the set of $K$-rational points of $X$. Similarly, $X\left(K_{v}\right)$ is the set of $K_{v}$-rational points of $X \otimes_{K} K_{v}$. Define $\mathbf{A}^{2}(K)$ and $\mathbf{A}^{2}\left(K_{v}\right)$ in the same way and equip all these spaces with the topology induced by $v$. Then $X\left(K_{v}\right)$ is compact and contains $X(K)$ as a dense subset. Further, $\mathbf{A}^{2}(K)$ is dense in $X(K)$, hence also in $X\left(K_{v}\right)$. This follows since every point in $X$ admits a Zariski open neighborhood $U$ isomorphic to affine 2-space such that $U \backslash \mathbf{A}^{2}$ is a proper Zariski closed subset.

2.5. Heights. See [Sil07, Section 3.1] or [Lan83, Section 3] for an introduction to heights. Let $K$ be a number field. For any absolute value $v \in M_{K}$ define a local height $\tau_{v}: \mathbf{A}^{2}(K) \rightarrow \mathbf{R}_{+}$by

$$
\tau_{v}=\log ^{+} \max \left\{\left|x_{1}\right|_{v},\left|x_{2}\right|_{v}\right\}=\log \max \left\{1,\left|x_{1}\right|_{v},\left|x_{2}\right|_{v}\right\}
$$

and define the (absolute, global) logarithmic height $h=h_{K}: \mathbf{A}^{2}(K) \rightarrow \mathbf{R}_{+}$by

$$
h=\sum_{v \in M_{K}} \frac{\left[K_{v}: \mathbf{Q}_{v}\right]}{[K: \mathbf{Q}]} \tau_{v} .
$$

If $L / K$ is a finite extension, then $h_{L}=h_{K}$ on $\mathbf{A}^{2}(K)$, see [Sil07, Proposition 3.4].

Let us express the local height $\tau_{v}$ in local coordinates at infinity. Consider an admissible compactification $X$ of $\mathbf{A}^{2}$ and a closed point $\xi \in X \backslash \mathbf{A}^{2}$. Let $L \supseteq K$ be a number field over which $X$ and $\xi$ are defined and extend $v$ as an absolute value on $L$.

\footnotetext{
${ }^{2}$ The inequality (2.3) is reversed in [FJ11] but this is a typo. The right-hand side of (2.3) is negative.
} 
Pick local coordinates $z_{i} \in \mathcal{O}_{X, \xi}, i=1,2$, defined over $L$ such that $X \backslash \mathbf{A}^{2} \subseteq E_{1} \cup E_{2}$, locally at $\xi$, where $E_{i}=\left\{z_{i}=0\right\}$. Given $\delta_{i}>0$ define

$$
\Omega_{v, \delta}:=\left\{\left.P \in \mathbf{A}^{2}\left(L_{v}\right)|| z_{i}(P)\right|_{v}<\delta_{i}, i=1,2\right\} .
$$

By Section 2.4 we have $\Omega_{v, \delta} \cap \mathbf{A}^{2}(L) \neq \emptyset$. It follows from (2.1) that if $0<\delta_{i} \ll 1$, then

$$
\tau_{v}=-\sum_{i=1}^{2} b_{i} \log \left|z_{i}\right|_{v}+O(1)
$$

on $\Omega_{v, \delta}$. Similarly, it follows from (2.2) that if $f: \mathbf{A}^{2} \rightarrow \mathbf{A}^{2}$ is a dominant polynomial mapping defined over $L$, then

$$
\tau_{v} \circ f \leq \sum_{i=1}^{2} \operatorname{ord}_{E_{i}}\left(f^{*} \ell\right) \log \left|z_{i}\right|_{v}+O(1)
$$

on $\Omega_{v, \delta}$, where $\ell \in \mathcal{L}$ is a general affine function.

2.6. Canonical height and arithmetic degree. Consider a dominant polynomial mapping $f: \mathbf{A}^{2} \rightarrow \mathbf{A}^{2}$ defined over $\overline{\mathbf{Q}}$. For simplicity assume $\lambda_{1}=\lambda_{1}(f)>1$. Silverman defines the arithmetic degree on $\mathbf{A}^{2}(\overline{\mathbf{Q}})$ by

$$
\alpha:=\alpha_{f}:=\limsup _{n \rightarrow \infty}\left(h \circ f^{n}\right)^{1 / n}
$$

and shows that $\alpha \leq \lambda_{1}$, see [Sil11, Proposition 12].

By Theorem 2.2 we have $\operatorname{deg}\left(f^{n}\right) \sim n^{l} \lambda_{1}^{n}$, where $l \in\{0,1\}$. Further, $l=0$ when $\lambda_{2}<\lambda_{1}^{2}$. Silverman defines the canonical height on $\mathbf{A}^{2}(\overline{\mathbf{Q}})$ by

$$
\hat{h}:=\hat{h}_{f}:=\lim \sup \frac{1}{n^{l} \lambda_{1}^{n}} h \circ f^{n}
$$

and shows in [Sil11, Proposition $19(\mathrm{~d})]$ that $\alpha(P)<\lambda_{1}$ implies $\hat{h}(P)=0$. Inspired by Silverman's work, we state

Conjecture 2.4. We have $\hat{h}(P)<\infty$ for all $P \in \mathbf{A}^{2}(\overline{\mathbf{Q}})$. Moreover, if $\lambda_{2}<\lambda_{1}^{2}$, then $\hat{h}(P)=0$ implies $\alpha(P)<\lambda_{1}$.

The second part of this conjecture would provide a converse to Proposition 19 (d) in [Sil11], whereas the first part would give an affirmative answer to a special case of Question 18 in loc. cit.

Our Main Theorem gives a positive answer to Conjecture 2.4 when $\lambda_{2}<\lambda_{1}$ and shows that, under this assumption, the limsup in the definition of $\hat{h}$ is in fact a limit. On the other hand, the following example shows that the last statement in Conjecture 2.4 may fail when $\lambda_{2}=\lambda_{1}^{2}$.

Example 2.5. [Sil11, Example 16]. If $f(x, y)=\left(x^{2}, x y^{2}\right)$, then $\lambda_{1}=2, \lambda_{2}=4$ and $\operatorname{deg} f^{n}=(n+2) \cdot 2^{n-1}$, so $l=1$. For $P=(2,0)$ we have $h\left(f^{n}(P)\right)=2^{n} \log 2$. In particular, $\hat{h}(P)=0$ but $\alpha(P)=\lambda_{1}=2$. 
See also [KS12] for definitions and results of canonical heights and arithmetic degrees for rational selfmaps of more general algebraic varieties.

\section{Proof of the main theorem}

Fix a polynomial mapping $f: \mathbf{A}^{2} \rightarrow \mathbf{A}^{2}$ defined over $\overline{\mathbf{Q}}$ and of small topological degree: $\lambda_{2}<\lambda_{1}$. Let $K_{0}=K_{0}(f)$ be a number field over which $f$ is defined.

\subsection{Growth of local heights.}

Proposition 3.1. We may choose $K_{0}$ above such that if $K \supseteq K_{0}$ and $v \in M_{K}$ is any normalized absolute value, then:

(i) the limit $\hat{\tau}_{v}:=\lim _{n \rightarrow \infty} \lambda_{1}^{-n} \tau_{v} \circ f^{n}$ exists, and is finite, pointwise on $\mathbf{A}^{2}(K)$;

(ii) the set $U_{v}^{+}:=\left\{\hat{\tau}_{v}>0\right\} \subseteq \mathbf{A}^{2}(K)$ is open and nonempty;

(iii) $\limsup _{n \rightarrow \infty}\left(\tau_{v} \circ f^{n}\right)^{1 / n} \leq \lambda_{2}$ on $\mathbf{A}^{2}(K) \backslash U_{v}^{+}$.

To prove this, pick $0<\varepsilon<\lambda_{1}-\lambda_{2}$. Given this $\varepsilon$, let $X, \xi_{+}$and $n_{0}$ be as in Theorem 2.3 and choose a number field $L=L(\varepsilon) \supseteq K$ such that $f$ and $X$ are defined over $L$ and the closed point $\xi_{+}$is $L$-rational. Extend $v$ as an absolute value on $L$.

Lemma 3.2. There exists an open subset $V_{v}^{+} \subseteq \mathbf{A}^{2}\left(L_{v}\right)$ such that $f\left(V_{v}^{+}\right) \subseteq V_{v}^{+}$and

(i) the limit $\hat{\tau}_{v}:=\lim _{n \rightarrow \infty} \lambda_{1}^{-n} \tau_{v} \circ f^{n}$ exists pointwise on $V_{v}^{+}$and $0<\hat{\tau}_{v}<\infty$;

(ii) $\mathbf{A}^{2}(L) \cap V_{v}^{+} \neq \emptyset$;

(iii) $\tau_{v} \circ f^{n_{0}} \leq\left(\lambda_{2}+\varepsilon\right)^{n_{0}} \tau_{v}+O(1)$ on $\mathbf{A}^{2}\left(L_{v}\right) \backslash V_{v}^{+}$, with $n_{0}$ as in Theorem 2.3.

This result is an analogue of [FJ11, Lemma 7.2], which in turn is modeled on results for the complex Hénon map. See also [Ing11, Lemma 2.1].

Granted Lemma 3.2 we conclude the proof of Proposition 3.1 as follows. Set

$$
U_{v}^{+}:=\mathbf{A}^{2}(K) \cap \bigcup_{n \geq 0} f^{-n}\left(V_{v}^{+}\right)
$$

By Lemma 3.2 (i), the limit defining $\hat{\tau}_{v}$ exists on the open set $U_{v}^{+}$and $0<\hat{\tau}_{v}<\infty$ there. Now suppose $P \in \mathbf{A}^{2}(K) \backslash U_{v}^{+}$. Since $f^{j n_{0}}(P) \notin V_{v}^{+}$for all $j \geq 0$, Lemma 3.2 (iii) yields $\lim \sup _{j \rightarrow \infty}\left(\tau_{v}\left(f^{j n_{0}}(P)\right)\right)^{1 / j n_{0}} \leq \lambda_{2}+\varepsilon$. Using the bound $\tau_{v} \circ f \leq(\operatorname{deg} f) \tau_{v}+$ $O(1)$ on $\mathbf{A}^{2}(K)$ (see [Sil07, (3.6) p. 92]) we obtain

$$
\limsup _{n \rightarrow \infty} \tau_{v}\left(f^{n}(P)\right)^{1 / n} \leq \lambda_{2}+\varepsilon<\lambda_{1} .
$$

Thus $\hat{\tau}_{v}$ is well defined and finite everywhere on $\mathbf{A}^{2}(K)$ and the set $U_{v}^{+}$is characterized, independently of $L$, as $U_{v}^{+}=\left\{\hat{\tau}_{v}>0\right\}$. Letting $\varepsilon \rightarrow 0$ we obtain (iii).

It only remains to prove that $U_{v}^{+}$is nonempty, assuming that $K_{0}$ is well chosen and $K \supseteq K_{0}$. For this, it suffices to consider the case $K=K_{0}$. Fix $\varepsilon_{0}$ with $0<\varepsilon_{0}<\lambda_{1}-\lambda_{2}$, apply Theorem 2.3 with $\varepsilon=\varepsilon_{0}$ and pick $K_{0}$ so that all the relevant data in that theorem (as well as $f$ ) are defined over $K_{0}$. Given $v \in M_{K_{0}}$, we now apply Lemma 3.2 with $L=K=K_{0}$. We obtain $U_{v}^{+} \supseteq \mathbf{A}^{2}\left(K_{0}\right) \cap V_{v}^{+} \neq \emptyset$ as desired.

Proof of Lemma 3.2. We first analyze the situation locally at infinity near the fixed point $\xi_{+}$. Pick local coordinates $\left(z_{1}, z_{2}\right)$ at $\xi_{+}$such that $X \backslash \mathbf{A}^{2} \subseteq E_{1} \cup E_{2}$, locally at $\xi$, where $E_{i}=\left\{z_{i}=0\right\}$. Given $0<\delta_{i} \ll 1$ define $\Omega_{v, \delta}$ as in (2.4). We claim that for 
suitable choices of $\delta_{i}$ we have $f\left(\Omega_{v, \delta}\right) \subseteq \Omega_{v, \delta}$ and that $\lambda_{1}^{-n} \tau_{v} \circ f^{n}$ converges pointwise on $\Omega_{v, \delta}$ to a strictly positive function $\hat{\tau}_{v}$. To see this, we consider the two cases (a) and (b) in Theorem 2.3 (3) separately.

In case (b) we have $E_{1} \cap \mathbf{A}^{2}=\emptyset \neq E_{2} \cap \mathbf{A}^{2}$ and

$$
f^{*} z_{1}=z_{1}^{\lambda_{1}} \phi_{1} \quad \text { and } \quad f^{*} z_{2}=z_{1} \phi_{2},
$$

where $\phi_{i} \in \mathcal{O}_{X, \xi_{+}}$and $\phi_{1}\left(\xi_{+}\right) \neq 0$. For $0<\delta_{1} \ll \delta_{2} \ll 1$ it follows, using $\lambda_{1} \geq 2$, that $f\left(\Omega_{v, \delta}\right) \subseteq \Omega_{v, \delta}$. We claim that as $n \rightarrow \infty$, the functions $s_{n}:=\lambda_{1}^{-n} \log \left|f^{n *} z_{1}\right|_{v}$ converge pointwise on $\Omega_{v, \delta}$ to a function $s_{\infty}<0$. Indeed, (3.2) yields $s_{n+1}=s_{n}+$ $\lambda_{1}^{-(n+1)} \log \left|\phi_{1} \circ f^{n}\right|_{v}$ and hence

$$
s_{n} \rightarrow s_{\infty}:=\log \left|z_{1}\right|_{v}+\sum_{0}^{\infty} \lambda_{1}^{-(j+1)} \log \left|\phi_{1} \circ f^{j}\right|_{v} \quad \text { as } n \rightarrow \infty .
$$

Here the series is uniformly bounded on $\Omega_{v, \delta}$, so $s_{\infty}<0$. Clearly $s_{\infty} \circ f=\lambda_{1} s_{\infty}$. Using (2.5) we can rewrite this in terms of the local height. Note that $b_{1}>0=b_{2}$ since $E_{1} \cap \mathbf{A}^{2}=\emptyset \neq E_{2} \cap \mathbf{A}^{2}$. Thus $\tau_{v}=-b_{1} \log \left|z_{1}\right|_{v}+O(1)$ on $\Omega_{v, \delta}$. It is then clear that $\lambda_{1}^{-n} \tau_{v} \circ f^{n}$ converges pointwise on $\Omega_{v, \delta}$ to the function $\hat{\tau}_{v}:=-b_{1} s_{\infty}>0$.

In case (a) we instead have $E_{i} \cap \mathbf{A}^{2}=\emptyset$ for $i=1,2$ and

$$
f^{*} z_{i}=z_{1}^{a_{i 1}} z_{2}^{a_{i 2}} \phi_{i}
$$

for $i=1,2$, where $a_{i j} \in \mathbf{N}, \phi_{i} \in \mathcal{O}_{X, \xi_{+}}$and $\phi_{i}\left(\xi_{+}\right) \neq 0$. The matrix $\left(a_{i j}\right)$ has irrational spectral radius $\lambda_{1}$ and hence admits an eigenvector $\zeta=\left(\zeta_{1}, \zeta_{2}\right)$ with $\zeta_{i}>0$. Set $\delta_{i}=\kappa^{\zeta_{i}}$ with $0<\kappa \ll 1$. Since $\lambda_{1}>1$ it follows that $f\left(\Omega_{v, \delta}\right) \subseteq \Omega_{v, \delta}$. We have

$$
f^{n *} z_{i}=z_{1}^{a_{i 1}^{(n)}} z_{2}^{a_{i 2}^{(n)}} \prod_{l=0}^{n-1}\left(\phi_{1}^{a_{i 1}^{(l)}} \phi_{2}^{a_{i 2}^{(l)}}\right) \circ f^{n-1-l},
$$

where, for $0 \leq l \leq n, a_{i j}^{(l)}$ are the entries in the matrix $A^{l}$. By the Perron-Frobenius Theorem, $\lambda_{1}^{-n} A^{n}$ converges as $n \rightarrow \infty$ to a matrix with strictly positive coefficients. Using (3.4) we see that for $i=1,2$, the sequence $\left(s_{i, n}\right)_{n=1}^{\infty}$ of functions on $\Omega_{v, \delta}$, defined by $s_{i, n}:=\lambda_{1}^{-n} \log \left|f^{n *} z_{i}\right|_{v}$, converges pointwise to a strictly negative function $s_{i, \infty}$. Clearly $s_{i, \infty} \circ f=\lambda_{1} s_{i, \infty}$. Using (2.5), this implies that $\lambda_{1}^{-n} \tau_{v} \circ f^{n}$ converges pointwise on $\Omega_{v, \delta}$ to the function $\hat{\tau}_{v}:=-\sum_{i=1}^{2} b_{i} s_{i, \infty}$. Since $b_{i}>0$ we again have $\hat{\tau}_{v}>0$.

Set $V_{v}^{+}:=f^{-n_{0}}\left(\Omega_{v, \delta}\right)$. Clearly $V_{v}^{+}$is open in $\mathbf{A}^{2}\left(L_{v}\right)$ and $f\left(V_{v}^{+}\right) \subseteq V_{v}^{+}$. Further, $\lambda_{1}^{-n} \tau_{v} \circ f^{n}$ converges pointwise on $V_{v}^{+}$to a function $\hat{\tau}_{v}>0$. By Section 2.4, we have $V_{v}^{+} \cap \mathbf{A}^{2}(L) \supseteq \Omega_{v, \delta} \cap \mathbf{A}^{2}(L) \neq \emptyset$.

It remains to prove the estimate in (iii). Let $\tilde{V}_{v}^{+} \subseteq X\left(L_{v}\right)$ be an open set such that $\mathbf{A}^{2}\left(L_{v}\right) \cap \tilde{V}_{v}^{+}=V_{v}^{+}$and $Z^{+}\left(L_{v}\right) \subseteq \tilde{V}_{v}^{+}$. Such a set can be constructed as follows. Following (2.4) set

$$
\tilde{\Omega}_{v, \delta}:=\left\{\left.P \in X\left(L_{v}\right)|| z_{i}(P)\right|_{v}<\delta_{i}, i=1,2\right\},
$$


so that $\tilde{\Omega}_{v, \delta} \cap \mathbf{A}^{2}\left(L_{v}\right)=\Omega_{v, \delta}$. Recall that the extension of $f^{n_{0}}$ as a rational selfmap of $X$ is regular at every point of $Z^{+}$and that $f^{n_{0}}\left(Z^{+}\right)=\left\{\xi^{+}\right\}$. We can therefore set

$$
\tilde{V}_{v}^{+}:=\left(f^{n_{0}}\right)^{-1}\left(\tilde{\Omega}_{v, \delta}\right) \backslash I
$$

where $I$ is the indeterminacy set of $f^{n_{0}}: X \rightarrow X$.

In order to prove (iii) it suffices, by compactness, to prove the estimate

$$
\tau_{v} \circ f^{n_{0}} \leq\left(\lambda_{2}+\varepsilon\right)^{n_{0}} \tau_{v}+O(1)
$$

in a neighborhood of any point $\eta \in X\left(L_{v}\right) \backslash \tilde{V}_{v}^{+}$. This is clear if $\eta \in \mathbf{A}^{2}\left(L_{v}\right)$, so we may assume $\eta \notin \mathbf{A}^{2}\left(L_{v}\right)$. Then $\eta \in Z^{-}\left(L_{v}\right) \backslash Z^{+}\left(L_{v}\right)$. Pick local coordinates $\left(w_{1}, w_{2}\right)$ at $\eta$, defined over $L_{v}$, such that $X\left(L_{v}\right) \backslash \mathbf{A}^{2}\left(L_{v}\right) \subseteq F_{1}\left(L_{v}\right) \cup F_{2}\left(L_{v}\right)$ locally at $\eta$, where $F_{i}=\left\{w_{i}=0\right\}$. Let $\tilde{G}_{\eta}$ be a small neighborhood of $\eta \in X\left(L_{v}\right)$. On $\tilde{G}_{\eta} \cap \mathbf{A}^{2}\left(L_{v}\right)$ we then have

$$
\begin{aligned}
\tau_{v} \circ f^{n_{0}} & \leq \sum_{i=1}^{2} \operatorname{ord}_{F_{i}}\left(f^{n_{0} *} \ell\right) \log \left|w_{i}\right|_{v}+O(1) \\
& \leq\left(\lambda_{2}+\varepsilon\right)^{n_{0}} \sum_{i=1}^{2} \operatorname{ord}_{F_{i}}(\ell) \log \left|w_{i}\right|_{v}+O(1) \\
& \leq\left(\lambda_{2}+\varepsilon\right)^{n_{0}} \tau_{v}+O(1)
\end{aligned}
$$

where $\ell \in \mathcal{L}$ is a general affine function. Here the first and third inequality follow from (2.6) and (2.5), respectively. The second inequality results from (2.3) when $F_{i} \subseteq$ $Z^{-}$and is trivial otherwise, since $\operatorname{ord}_{F_{i}}\left(f^{*} \ell\right) \leq 0=\operatorname{ord}_{F_{i}}(\ell)$ in this case.

Thus (3.5) holds, which completes the proof.

3.2. Growth of heights. Let $K_{0}$ be as in Proposition 3.1. Pick any point $P \in$ $\mathbf{A}^{2}(\overline{\mathbf{Q}})$ and let $K \supseteq K_{0}$ be a number field such that $P$ is $K$-rational. We claim that there exists a finite subset $M_{K}^{\prime}=M_{K}^{\prime}(P) \subseteq M_{K}$ such that

$$
\tau_{v}\left(f^{n}(P)\right)=0 \quad \text { for all } v \in M_{K} \backslash M_{K}^{\prime} \text { and all } n \geq 0 \text {. }
$$

To see this, note that $f^{*} x_{i} \in K\left[x_{1}, x_{2}\right], i=1,2$, so for all but finitely many $v \in M_{K}$ we will have $f^{*} x_{i} \in \mathfrak{o}_{v}\left[x_{1}, x_{2}\right]$, where $\mathfrak{o}_{v}=\left\{\left.a \in K|| a\right|_{v} \leq 1\right\}$. For these $v$ we then also have $f^{n *} x_{i} \in \mathfrak{o}_{v}\left[x_{1}, x_{2}\right]$ for all $n \geq 1$. Further, for all but finitely many $v$ we have $x_{i}(P) \in \mathfrak{o}_{v}$. Combining these statements and the definition of $\tau_{v}$, we obtain (3.6).

It is now easy to conclude. We have

$$
h\left(f^{n}(P)\right)=\sum_{v \in M_{K}} \frac{\left[K_{v}: \mathbf{Q}_{v}\right]}{[K: \mathbf{Q}]} \tau_{v}\left(f^{n}(P)\right)=\sum_{v \in M_{K}^{\prime}} \frac{\left[K_{v}: \mathbf{Q}_{v}\right]}{[K: \mathbf{Q}]} \tau_{v}\left(f^{n}(P)\right)
$$

so since $M_{K}^{\prime}$ is finite, all assertions in the Main Theorem follow from Proposition 3.1, except for the claim about polynomial automorphisms. 
3.3. Polynomial automorphisms. Now assume that $f$ is a polynomial automorphism with $\lambda_{1}>1$. We must prove that if $\hat{h}(P)=0$, then $P$ is periodic.

First suppose $f$ is regular [Sib99] in the sense that the indeterminacy loci of the birational maps $f, f^{-1}: \mathbf{P}^{2} \rightarrow \mathbf{P}^{2}$ are disjoint.

From Propositions 2.2.2 and 2.3.2 in [Sib99] we deduce the following facts: $\operatorname{deg} f=$ $\operatorname{deg} f^{-1}=\lambda_{1}$; the indeterminacy locus of $f$ (resp. $f^{-1}$ ) is a single point $\xi_{-}$(resp. $\left.\xi_{+}\right)$on the line at infinity $L_{\infty}=\mathbf{P}^{2} \backslash \mathbf{A}^{2}$ with $\xi_{+} \neq \xi_{-} ; f\left(L_{\infty} \backslash\left\{\xi_{-}\right\}\right)=\xi_{+}$and $f^{-1}\left(L_{\infty} \backslash\left\{\xi_{+}\right\}\right)=\xi_{-}$.

Let $X$ be the minimal admissible compactification of $\mathbf{A}^{2}$ for which the induced birational map $f: X \rightarrow \mathbf{P}^{2}$ is a morphism (i. e., without indeterminacy points). Concretely, $X$ is obtained from $\mathbf{P}^{2}$ by successively blowing up the indeterminacy locus of $f$. Since $f$ is birational, $X \backslash \mathbf{A}^{2}$ has a unique irreducible component $E_{-}$that is mapped onto $L_{\infty}$ by $f$. All other irreducible components are mapped to $\xi_{+}$.

Set $Z^{-}:=E_{-}$and $Z^{+}:=X \backslash\left(\mathbf{A}^{2} \cup E_{-}\right)$. We claim that a strong version of Theorem 2.3 holds, with $n_{0}=1$. Indeed, note that statement (2) holds. The same is true of statement (3): we are in case (b), with $E=L_{\infty}$. Further, we claim that

$$
\operatorname{ord}_{E_{-}}\left(f^{*} \ell\right)=-1 \quad \text { and } \quad \operatorname{ord}_{E_{-}}(\ell)=-\lambda_{1},
$$

for a general affine function $\ell$. Since $\lambda_{1}>\lambda_{2}=1$, this is stronger than (1).

To prove (3.7), recall that $f\left(E_{-}\right)=L_{\infty}$. Since $f$ is an automorphism, this implies $\operatorname{ord}_{E_{-}}\left(f^{*} \ell\right)=\operatorname{ord}_{L_{\infty}}(\ell)=-1$. Similarly, $\operatorname{ord}_{E_{-}}(\ell)=\operatorname{ord}_{L_{\infty}}\left(\left(f^{-1}\right)^{*} \ell\right)=-\operatorname{deg} f^{-1}=$ $-\lambda_{1}$.

Fix a number field $K_{0}$ over which $f$ and $X$ are defined. Suppose $K \supseteq K_{0}$ and consider an absolute value $v \in M_{K}$. Using (3.7) we prove Lemma 3.2 with $L=K$ and the following estimate, which is stronger than the one in (iii):

$$
\tau_{v} \circ f \leq \lambda_{1}^{-1} \tau_{v}+O(1)
$$

on $\mathbf{A}^{2}\left(K_{v}\right) \backslash V_{v}^{+}$. Since $\lambda_{1}>1$, it follows that the sequence $\left(\tau_{v} \circ f^{n}\right)_{n=0}^{\infty}$ must be pointwise bounded on $\mathbf{A}^{2}(K) \backslash U_{v}^{+}$, where $U_{v}^{+}$is defined as in (3.1).

Now fix $P \in \mathbf{A}^{2}(\overline{\mathbf{Q}})$ with $\hat{h}(P)=0$. Pick $K \supseteq K_{0}$ such that $P \in \mathbf{A}^{2}(K)$. From the preceding paragraph and the arguments in Section 3.2 it follows that the sequence $\left(h\left(f^{n}(P)\right)\right)_{n=0}^{\infty}$ is bounded, which by the Northcott Theorem implies that $P$ is preperiodic, hence periodic.

Finally we treat the general case when $f$ is not necessarily regular. It follows from [FM89] that there exists a polynomial automorphism $g: \mathbf{A}^{2} \rightarrow \mathbf{A}^{2}$ such that $\tilde{f}:=$ $g^{-1} f g$ is regular. Set $D:=(\operatorname{deg} g)\left(\operatorname{deg} g^{-1}\right)$. Since $f^{n}=g \tilde{f}^{n} g^{-1}$ we have $D^{-1} \operatorname{deg} \tilde{f}^{n} \leq$ $\operatorname{deg} f^{n} \leq D \operatorname{deg} \tilde{f}^{n}$ and hence $\lambda_{1}(\tilde{f})=\lambda_{1}(f)$.

As for the growth of heights, we already know that the limits

$$
\hat{h}_{f}:=\lim _{n \rightarrow \infty} \lambda_{1}^{-n} h \circ f^{n} \quad \text { and } \quad \hat{h}_{\tilde{f}}:=\lim _{n \rightarrow \infty} \lambda_{1}^{-n} h \circ \tilde{f}^{n}
$$

exist, pointwise on $\mathbf{A}^{2}(\overline{\mathbf{Q}})$. Since $\operatorname{deg} g^{ \pm 1} \leq D$, we have by [Sil07, Theorem 3.11] that

$$
D^{-1}\left(h \circ \tilde{f}^{n}\right)+O(1) \leq h \circ f^{n} \circ g \leq D\left(h \circ \tilde{f}^{n}\right)+O(1)
$$


and hence $D^{-1} \hat{h}_{\tilde{f}} \leq \hat{h}_{f} \circ g \leq D \hat{h}_{\tilde{f}}$. In particular, if $\hat{h}_{f}(P)=0$, then $\hat{h}_{\tilde{f}}\left(g^{-1}(P)\right)=0$. By what precedes, $g^{-1}(P)$ is then periodic under $\tilde{f}$, so that $P$ is periodic under $f$. This concludes the proof of the Main Theorem.

3.4. A noninvertible example. Let us consider the map

$$
f(x, y)=\left(y^{2}(x y+1), x\left(x y^{3}+1\right)\right) .
$$

The topological degree is $\lambda_{2}=4$. For example, the point $(0,0)$ has three preimages $(0,0),(1,-1)$ and $(-1,1)$, with multiplicities 2,1 and 1 , respectively. It is easy to find a recursion relation for $\left(\operatorname{deg} f^{n}\right)_{n=0}^{\infty}$ and show that the first dynamical degree is $\lambda_{1}=2+\sqrt{7}$. In particular, $\lambda_{1}>\lambda_{2}$, so $f$ is of small topological degree.

Note that $f^{2}(x, 0)=\left(x^{2}, 0\right)$ and $f^{2}(0, y)=\left(0, y^{2}\right)$. Since $\lambda_{1}>\sqrt{2}$, the coordinate axes are contained in the locus $\{\hat{h}=0\}$, which therefore contains points for which $h \circ f^{n}$ grows exponentially.

\section{Acknowledgment}

We thank Dennis Eriksson, Shu Kawaguchi, Joey Lee, Mircea Mustaţă and Joe Silverman for fruitful discussions. The first author was supported by the NSF and the second author by the Swedish Research Council.

\section{References}

[BS91] E. Bedford and J. Smillie, Polynomial diffeomorphisms of $\mathbf{C}^{2}$ : currents, equilibrium measure and hyperbolicity, Invent. Math. 103 (1991), 69-99.

[BFJ08] S. Boucksom, C. Favre and M. Jonsson, Degree growth of meromorphic surface maps, Duke Math. J. 141 (2008), 519-538

[Den95] L. Denis, Points périodiques des automorphismes affines, J. Reine Angew. Math. 467 (1995), 157-167.

[DDG1] J. Diller, R. Dujardin and V. Guedj, Dynamics of meromorphic maps with small topological degree I: from cohomology to currents, Indiana Univ. Math. J. 59 (2010), 521-562.

[DDG2] J. Diller, R. Dujardin and V. Guedj, Dynamics of meromorphic maps with small topological degree II: Energy and invariant measure, Comment. Math. Helv. 86 (2011), 277-316.

[DDG3] J. Diller, R. Dujardin and V. Guedj, Dynamics of meromorphic maps with small topological degree III: geometric currents and ergodic theory, Ann. Sci. École Norm. Sup. 43 (2010), 235-278.

[Fav00] C. Favre, Classification of 2-dimensional contracting rigid germs and Kato surfaces. I, J. Math. Pures Appl. 79 (2000), 475-514.

[FJ04] C. Favre and M. Jonsson, The valuative tree, Lecture Notes in Mathematics, vol 1853. Springer, 2004.

[FJ07] C. Favre and M. Jonsson, Eigenvaluations, Ann. Sci. École Norm. Sup. 40 (2007), 309-349.

[FJ11] C. Favre and M. Jonsson, Dynamical compactifications of $\mathbf{C}^{2}$, Ann. Math. 173 (2011), 211-249.

[FS92] J.-E. Fornæss and N. Sibony, Complex Hénon mappings in $\mathbf{C}^{2}$ and Fatou-Bieberbach domains, Duke Math. J. 65(2) (1992), 345-380.

[FM89] S. Friedland and J. Milnor, Dynamical properties of plane polynomial automorphisms, Ergodic Theory Dynam. Systems 9 (1989), 67-69.

[Gue02] V. Guedj, Dynamics of polynomial mappings of $\mathbf{C}^{2}$, Amer. J. Math. 124 (2002), 75-106.

[Hub86] J. Hubbard, The Hénon mapping in the complex domain, In Chaotic dynamics and fractals (Atlanta, Ga., 1985). Notes Rep. Math. Sci. Engrg. 2, 101-111. Academic Press, Orlando, FL, 1986.

[HO94] J. Hubbard and R. Oberste-Vorth, Hénon mappings in the complex domain. I. The global topology of dynamical space, Inst. Hautes Études Sci. Publ. Math. 79 (1994), 5-46. 
[Ing11] P. Ingram, Canonical heights for Hénon maps, arXiv:1111.3609v1.

[Jon12] M. Jonsson. Dynamics on Berkovich spaces in low dimensions, arXiv:1201.1944. To appear in Berkovich spaces and applications, Séminaires et Congrès, Société Mathématique de France.

[Kaw06] S. Kawaguchi, Canonical height functions for affine plane automorphisms, Math. Ann. 335 (2006), 285-310.

[Kaw09] S. Kawaguchi, Local and global canonical height functions for affine space automorphisms, To appear in Algebra Number Theory. arXiv:0909.3573.

[KS12] S. Kawaguchi and J.H. Silverman, On the dynamical and arithmetic degrees of rational self-maps of algebraic varieties, arXiv:1208.0815.

[Lan83] S. Lang, Fundamentals of Diophantine geometry, Springer-Verlag, New York, 1983.

[Lee09] C.-G. Lee, An upper bound for the height for regular affine automorphisms of $\mathbf{A}^{n}$, Math. Ann. 355 (2013), 1-16.

[Mar00] S. Marcello, Sur les propriétés arithmétiques des itérés d'automorphismes réguliers, C. R. Acad. Sci. Paris Sér. I Math. 331 (2000), 11-16.

[Mar03] S. Marcello, Sur la dynamique arithmétique des automorphismes de l'espace affine, Bull. Soc. Math. France 131 (2003), 229-257.

[Sib99] N. Sibony, Dynamique des applications rationnelles de $\mathbf{P}^{k}$, In Dynamique et géométrie complexes (Lyon, 1997), Panor. Synthèses, 8, 97-185. Soc. Math. France, Paris, 1999.

[Sil94] J.H. Silverman, Geometric and arithmetic properties of the Hénon map, Math. Z. 215 (1994), 237-250.

[Sil07] J.H. Silverman, The arithmetic of dynamical systems, Graduate Texts in Mathematics, volume 241. Springer, New York, 2007.

[Sil11] J.H. Silverman, Dynamical degrees, arithmetic entropy, and canonical heights for dominant rational self-maps of projective space, arXiv:1111.5664v2. To appear in Ergodic Theory Dynam. Systems.

Department of Mathematics, University of Michigan, Ann Arbor, Mi 48109-1043, USA

E-mail address: mattiasj@umich.edu

Department of Mathematics, Chalmers University of Technology and the University of Gothenburg, SE-412 96 Göteborg, Sweden

E-mail address: wulcan@chalmers.se 
